\title{
Pleckstrin 2 is a potential drug target for colorectal carcinoma with activation of $\mathrm{APC} / \boldsymbol{\beta}$-catenin
}

\author{
BAO CHAI ${ }^{1}$, YARONG GUO $^{2}$, NA ZHU $^{1}$, JUNMEI JIA ${ }^{2}$, ZHUOWEI ZHANG $^{3}$, \\ MEI PING ${ }^{2}$, KAI JIA ${ }^{4}$, XIAOLONG CUI ${ }^{4}$ and YUHONG SUO ${ }^{5}$
}

\begin{abstract}
${ }^{1}$ Department of Gastroenterology, Shanxi Academy of Medical Science, Shanxi Bethune Hospital, Taiyuan, Shanxi 030032; ${ }^{2}$ Department of Oncology, The First Affiliated Hospital of Shanxi Medical University, Taiyuan, Shanxi 030001; ${ }^{3}$ Medical Imaging Department, Shanxi Medical University, Taiyuan, Shanxi 030031;

${ }^{4}$ Department of Surgery, The First Affiliated Hospital of Shanxi Medical University, Taiyuan, Shanxi 030031;

${ }^{5}$ Liver Cancer Center, Tianjin Medical University Cancer Institute and Hospital, Tianjin 300181, P.R. China
\end{abstract}

Received March 29, 2021; Accepted August 16, 2021

DOI: $10.3892 / \mathrm{mmr} .2021 .12502$

\begin{abstract}
The tumor suppressor gene adenomatous polyposis coli (APC) is frequently inactivated or absent in colorectal carcinoma (CRC). Loss-of-function of APC promotes the expression of $\beta$-catenin, which is critical for CRC development. Since $\beta$-catenin acts as an important transcription factor, blockage of $\beta$-catenin may have side effects, including impairment of tissue homeostasis and regeneration, thus limiting the application of $\beta$-catenin inhibitors for the treatment of patients with CRC. Therefore, identifying a novel substrate of APC $/ \beta$-catenin may provide essential clues to develop effective drugs. Small interfering RNA technology and lentivirus-mediated overexpression were performed for knockdown and overexpression of pleckstrin 2 (PLEK2) in CRC cells. Cell Counting Kit-8 and colony formation assays, and cell cycle analysis and cell apoptosis detection were used to detect the capacity of cell proliferation, cell cycle distribution and apoptosis. The present study demonstrated that the APC $/ \beta$-catenin signaling cascade transcriptionally activated PLEK2 in CRC cells. PLEK2 expression was markedly increased in CRC tissues. There was an inverse correlation between APC and PLEK2 expression in patients with CRC. In vitro, overexpression of PLEK2 increased the proliferation of CRC cells. Opposite results were observed in the cells with knockdown of PLEK2. Furthermore, PLEK2 promoted cell cycle progression and suppressed apoptosis. In summary, upregulation of PLEK2 contributed to CRC proliferation and colony formation activated by the APC $/ \beta$-catenin signal pathway. Targeting PLEK2 may be important for the treatment
\end{abstract}

Correspondence to: Dr Yarong Guo, Department of Oncology, The First Affiliated Hospital of Shanxi Medical University, 85 South Jiefang Road, Taiyuan, Shanxi 030001, P.R. China

E-mail: gyr5258@126.com

Key words: adenomatous polyposis coli, $\beta$-catenin, pleckstrin 2, colorectal carcinoma, proliferation of patients with $\mathrm{CRC}$ with activation of the APC/ $\beta$-catenin signaling pathway.

\section{Introduction}

According to the cancer statistics reported in 2021, an estimated 1.93 million new colorectal carcinoma (CRC) cases occurred in 2020, making it the third most common malignancy globally (1). Although the development of surgery, chemotherapy, radiotherapy, targeted therapy and immunotherapy has improved the treatment effectiveness for patients with CRC, the 5-year overall survival rate of patients is still poor $(2,3)$. Since the 1990s, 5 -fluorouracil (5-FU) or the 5-FU prodrug capecitabine has become the systemic treatment for CRC (4). Subsequently, the combination of 5-FU and leucovorin is the first-line treatment option for metastatic CRC, which can prolong the overall 2-year survival of the patients (4). However, more than half of the patients develop resistance to this treatment (5). Understanding the mechanism may help overcome drug resistance.

Development of CRC is driven by environmental exposure and genetic variants. Based on genomics, transcriptomics and proteomics studies, great progress has been made regarding the molecular insights of CRC (6). An inactivation mutation or downregulation of adenomatous polyposis coli (APC) has been found to contribute to approximately half of the cases of CRC $(7,8)$. When APC is normally expressed in cells, it interacts with $\beta$-catenin, and regulates GSK $3 \beta$-mediated phosphorylation of $\beta$-catenin, thus maintaining its protein stability (9). By contrast, loss of APC promotes the upregulation of $\beta$-catenin, which enters into the cell nucleus and exerts a transcriptional activation function (10). Based on these findings, inhibitors of Wnt/ $\beta$-catenin, such as LGK-974, are effective for the treatment of CRC in vitro and in a mouse model $(11,12)$. As mentioned in previous studies, several WNT ligand antagonists and porcupine inhibitors have been investigated in clinicals trials of WNT-associated human cancer types (13-15). However, blockage of Wnt/ $\beta$-catenin has not been applied for CRC treatment in clinical settings, mainly due to the side effects, including impairment of tissue homeostasis 
and regeneration, as well as the bioavailability of flavonoids during cellular metabolism, which need to be resolved (13). Therefore, it is urgent to investigate the downstream target of the Wnt/ $\beta$-catenin signaling cascade, which can promote CRC growth and metastasis.

Pleckstrin 2 (PLEK2) is a $40-\mathrm{kDa}$ protein which has been primarily identified as a substrate for protein kinase $\mathrm{C}$. It is widely expressed in multiple organs (16). Recent studies have demonstrated that overexpression of PLEK2 is associated with the development of cancer (17-19). The expression levels of PLEK2 are positively regulated by Janus kinase 2 (JAK2)/STAT5 and are elevated in philadelphia-chromosome-negative myeloproliferative neoplasms (MPNs) (20). Depletion of PLEK2 reverted lethality for MPNs tumorigenesis in JAK2V617F-knockin mice (21). Upregulation of PLEK2 has also been observed in gallbladder cancer (GBC) and serves a tumor-promoting role in GBC via regulation of the EGFR/C-C motif chemokine ligand 2 signaling pathway (17). In addition, high expression levels of PLEK2 are associated with the migration and invasion of non-small cell lung cancer (NSCLC) induced by TGF- $\beta 1$ (22). These results suggest that PLEK2 may serve a role in tumor growth and metastasis. However, to the best of our knowledge, the significance of PLEK2 in CRC remains unclear.

The present study aimed to investigate the connection between Wnt/APC/ $\beta$-catenin and PLEK2 and the role of PLEK2 in CRC growth and chemotherapy. 'Gain of function' and 'loss of function' strategies were employed to explore the biological functions of PLEK2 in CRC cell lines. It was demonstrated that activation of $\mathrm{Wnt} / \mathrm{APC} / \beta$-catenin signaling stimulated PLEK 2 expression, which was upregulated in CRC tissues. Gain and loss of function assay results revealed that PLEK2 was critical for CRC cell proliferation and apoptosis. Finally, PLEK2 overexpression conferred 5-FU resistance to CRC cells. Therefore, PLEK2 may be a novel target for the treatment of CRC with hyper-activated Wnt/APC/ $\beta$-catenin.

\section{Materials and methods}

Analysis of PLEK2 expression in patients with CRC based on The Cancer Genome Atlas (TCGA) database. Using TCGA (https://www.cancer.gov/about-nci/organization/ccg/research/structural-genomics/tcga),PLEK2expression in patients with CRC was analysed. To determine the clinical relevance of PLEK 2 in patients with CRC, the expression levels of PLEK2 were analyzed in cancer and normal samples using Gene Expression Profiling Interactive Analysis (GEPIA2021; http://gepia.cancer-pku.cn/) (23). A total of 257 colon adenocarcinoma samples and 349 normal tissues were included in the analysis.

Cell culture. Human normal colorectal cells (HIEC) and human CRC cells (RKO and HCT-116) were purchased from American Type Culture Collection. The cells were cultured in RPMI-1640 complete medium (Gibco; Thermo Fisher Scientific, Inc.), supplemented with 10\% FBS (Gibco; Thermo Fisher Scientific, Inc.) and 1\% penicillin-streptomycin solution (Corning, Inc.). The cell culture was kept in a cell incubator at $37^{\circ} \mathrm{C}$ with $5 \% \mathrm{CO}_{2}$. For the 5-FU treatment, cells were treated with different concentrations $(0,15.4,38.5,77,308$ and $616 \mu \mathrm{M})$ of 5-FU (cat. no. HY-90006; MedChemExpress) for $48 \mathrm{~h}$ at $37^{\circ} \mathrm{C}$. DMSO was used as a vehicle to dissolve 5-FU.

APC, catenin $\beta 1$ ( $\beta$-catenin) and PLEK2 knockdown using small interfering RNA (siRNA/si). siRNAs were synthesized by Huzhou Hippo Biotechnology Co., Ltd. siRNAs were dissolved in RNase-free water. Cells were seeded in 6-well plates at a density of 5x106 cells/well and then transfected with $10 \mathrm{nM}$ siRNAs (scrambled siCtrl, 5'-UUCUCCGAACGU GUCACGU-3'; siAPC, 5'-GGAAUCAACCCUCAAAAGU-3'; si $\beta$-catenin, 5'-CACTAACCAAGCTGAGTTT-3'; siPLEK2\#1, 5'-ACCUCUUCAAAGUGAUUACUA-3'; and SiPLEK2\#2, 5'-CCAGCUUUCCUGCAUUACUAU-3') using RNAiMAX (Invitrogen; Thermo Fisher Scientific, Inc.). Transfection was performed for $48 \mathrm{~h}$ at $37^{\circ} \mathrm{C}$. Total RNA and protein were harvested after $48 \mathrm{~h}$. Cellular function assays, including Cell Counting Kit-8 (CCK8), colony formation, apoptosis and cell cycle assays, were performed at $48 \mathrm{~h}$ after siRNA transfection.

PLEK2 overexpression. To overexpress PLEK2, the coding sequence of PLEK2 was cloned into pCDNA3.1 vectors (Beijing Tianyi Huiyuan Biotechnology Co., Ltd.). HCT-116 and RKO cells were seeded into 6-well plates at a density of $5 \times 10^{5}$ cells per well. After $12 \mathrm{~h}$, the cells were transfected with $5 \mu \mathrm{g}$ pCDNA3.1-Ctrl and pCDNA3.1-PLEK2 vectors using Lipofectamine ${ }^{\circledR} 2000$ (Invitrogen; Thermo Fisher Scientific, Inc.) for $48 \mathrm{~h}$ at $37^{\circ} \mathrm{C}$. Subsequently, PLEK 2 overexpression was checked using immunoblotting and the cells were subjected to cellular biology function assessment.

$R N A$ isolation and reverse transcription-quantitative PCR $(R T-q P C R)$. To detect mRNA expression, total RNA was isolated from CRC cells using TRIzol ${ }^{\circledR}$ reagent (Invitrogen; Thermo Fisher Scientific, Inc.) according to the manufacturer's protocol. mRNA was reverse transcribed into cDNA using the reverse transcriptional kit (Promega Corporation) according to the manufacturer's protocol. Subsequently, mRNA expression was quantified by detecting its cDNA abundance using SYBR Green master mixture (Qiagen, Inc.) on a Biorad qPCR system (Bio-Rad Laboratories, Inc.). The following steps were used for qPCR: Initial denaturation at $95^{\circ} \mathrm{C}$ for $5 \mathrm{~min}$; followed by 40 cycles of $95^{\circ} \mathrm{C}$ for $15 \mathrm{sec}, 60^{\circ} \mathrm{C}$ for $30 \mathrm{sec}$ and $70^{\circ} \mathrm{C}$ for $10 \mathrm{sec}$. The qPCR primer sequences were as follows: PLEK2 forward, 5'-CCGAAGCATGGGAGCCATT-3' and reverse, 5'-AGTGCTCAGGCTAATTTCTTCC-3'; $\beta$-catenin forward, 5'-AGCTTCCAGACACGCTATCAT-3' and reverse, 5'-CGG TACAACGAGCTGTTTCTAC-3'; and GAPDH forward, 5'-TGACTTCAACAGCGACACCCA-3' and reverse, 5'-CAC CCTGTTGCTGTAGCCAAA-3. GAPDH was used as an internal control. The $2^{-\Delta \Delta \mathrm{Cq}}$ method was used to calculate relative expression (24).

Immunoblotting assay. Protein was obtained from CRC cells using RIPA buffer (Beyotime Institute of Biotechnology) supplemented with protease inhibitor cocktail. The concentration of total protein was checked using a BCA assay kit (Beyotime Institute of Biotechnology). Nuclear fractions of the total protein were separated using NE-PER Nuclear and Cytoplasmic Extraction Reagent (Thermo Fisher Scientific, Inc.). Subsequently, proteins $(30 \mu \mathrm{g})$ were subjected to 
A

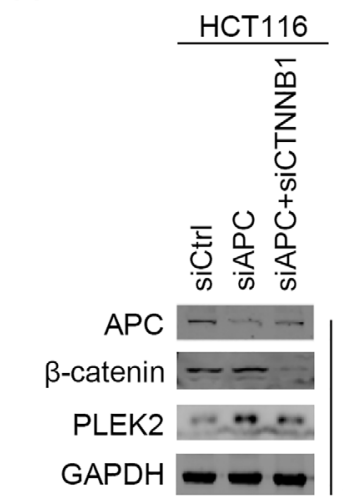

D

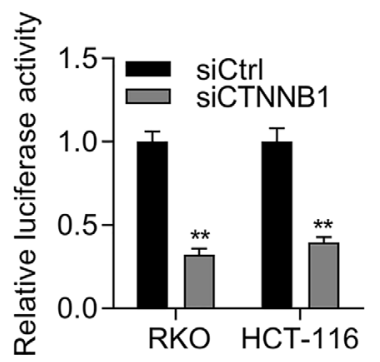

B

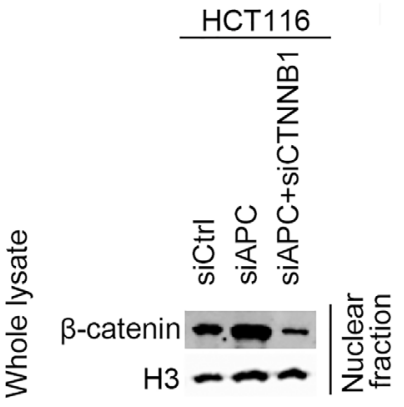

E

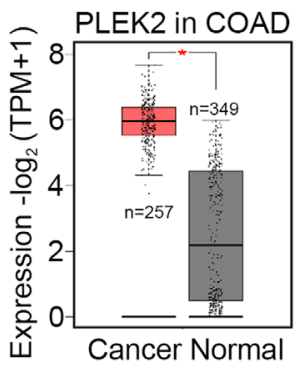

C

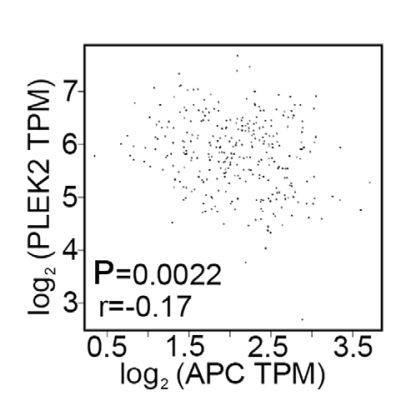

Figure 1. APC/ $\beta$-catenin activates PLEK2 in CRC cells and patients with CRC. (A) HCT-116 cells were transfected with siCtrl and siAPC. The cells were subjected to immunoblotting analysis of APC, $\beta$-catenin and PLEK2. (B) HCT-116 cells were transfected with siCtrl and siAPC. The nuclear extract was subjected to immunoblotting analysis of $\beta$-catenin. The expression levels of indicated genes were normalized to H3. (C) HCT-116 cells were transfected with siCtrl, siAPC or siAPC + $\beta$-catenin. The cells were subjected to reverse transcription-quantitative PCR analysis of PLEK2 expression. The expression levels of indicated genes were normalized to GAPDH. ${ }^{* *} \mathrm{P}<0.01$. (D) RKO and HCT-116 cells were transfected with pGL3. Basic vectors, which contained the PLEK2 promoter sequence, thymidine kinase TK vectors, as well as siCtrl or si $\beta$-catenin were co-transfected into RKO orHCT-116 cells. Dual luciferase activity was examined in RKO and HCT-116 cells transfected with siCtrl and si $\beta$-catenin. ${ }^{* *} \mathrm{P}<0.01$ vs. siCtrl. (E) Expression levels of PLEK2 were analyzed in CRC and normal tissues from TCGA. "P<0.05. (F) Spearman correlation between APC and PLEK2 expression in CRC tissues from TCGA. APC, adenomatous polyposis coli; COAD, colon adenocarcinoma; CRC, colorectal carcinoma; CTNNB1, catenin $\beta 1$; PLEK2, pleckstrin 2; si/siRNA, small interfering RNA; siCtrl, negative control siRNA; TCGA, The Cancer Genome Atlas; TPM, transcripts per million.

immunoblotting experiments according to the procedures described previously (25). Briefly, proteins were separated via $12 \%$ SDS-PAGE and then transferred to PVDF membranes. After transfection, the membranes were blocked with $5 \%$ skim milk for $2 \mathrm{~h}$ at room temperature. Then, the membranes were incubated with the primary antibodies at $4^{\circ} \mathrm{C}$ overnight. Subsequently, the membranes were incubated with the secondary antibodies for $2 \mathrm{~h}$ at room temperature. The following primary and secondary antibodies were used: PLEK2 (1:1,000; cat. no. ab121131; Abcam), APC (1:1,000; cat. no. 2504; Cell Signaling Technology, Inc.), $\beta$-catenin (1:1,000; cat. no. 8480; Cell Signaling Technology, Inc.), GAPDH (1:1,000; cat. no. 2118; Cell Signaling Technology, Inc.), Histone H3 (1:1,000; cat. no. 4499; Cell Signaling Technology, Inc.), anti-rabbit IgG HRP-conjugated (1:2,000; cat. no. 7074; Cell Signaling Technology, Inc.) and anti-mouse HRP-conjugated (1:2,000; cat. no. 7076; Cell Signaling Technology, Inc.). The proteins were detected using Pierce ${ }^{\mathrm{TM}}$ ECL Western Blotting substrate (Thermo Fisher Scientific, Inc.) and analyzed using Image Lab 6.1 software (Bio Rad Laboratories, Inc.).

CCK8 assay. The proliferation rate of $\mathrm{CRC}$ cells was determined using a CCK8 assay. In brief, 2,000 CRC cells were seeded into 96-well plates supplemented with $100 \mu \mathrm{l}$
RPMI-1640 complete medium. After 1, 2, 3 and 4 days, $10 \mu 1$ CCK8 (Beyotime Institute of Biotechnology) reagent was added into each well and the cells were incubated for $3 \mathrm{~h}$ in the cell incubator. Subsequently, the optical density value at $450 \mathrm{~nm}$ was measured to assess cell proliferation.

Colony formation. Colony formation was examined by seeding a density of $1.5 \times 10^{3} \mathrm{CRC}$ cells/well into 6-well plates. After transfection with siRNAs for $48 \mathrm{~h}$ at $37^{\circ} \mathrm{C}$, cell colonies were cultured for 10 days. Then the culture medium was removed, and the cells were washed with PBS, fixed with $100 \%$ methanol at room temperature for $15 \mathrm{~min}$ and stained with $0.2 \%$ crystal violet solution at room temperature for $30 \mathrm{~min}$. Images were captured using a camera (Nikon Corporation) to count the cell colonies (>50 cells) manually.

Cell cycle analysis. To detect the cell cycle distribution, the cells were first fixed with iced $70 \%$ ethanol overnight at $4^{\circ} \mathrm{C}$ and then stained with PI using the Cell Cycle and Apoptosis Analysis kit (Shanghai Yeasen Biotechnology Co., Ltd.) according to the manufacturer's instructions. Subsequently, cell cycle distribution was analyzed using a flow cytometry system (cytoFlex; Beckman Coulter, Inc.). Data were analyzed using Cytexpert software (version 2.4.0.28; Beckman Coulter, Inc.). 
A

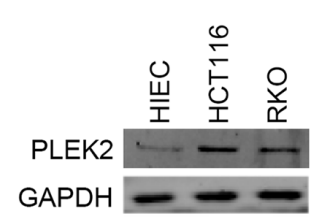

D

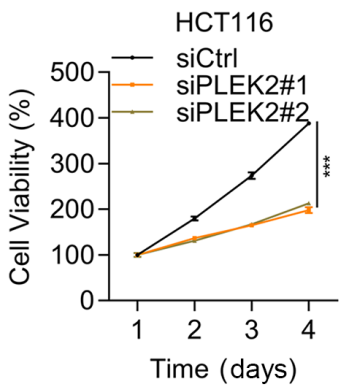

B

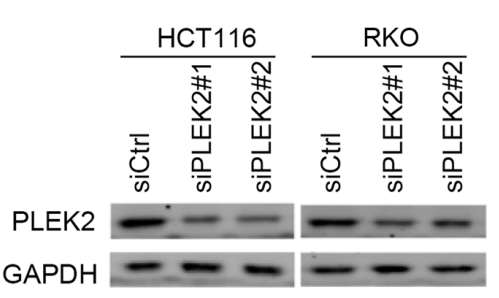

C

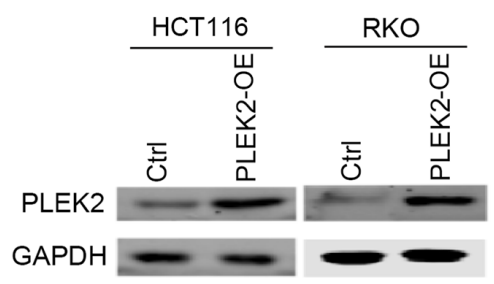

$\mathrm{F}$

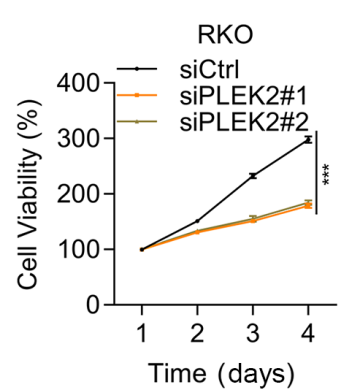

E
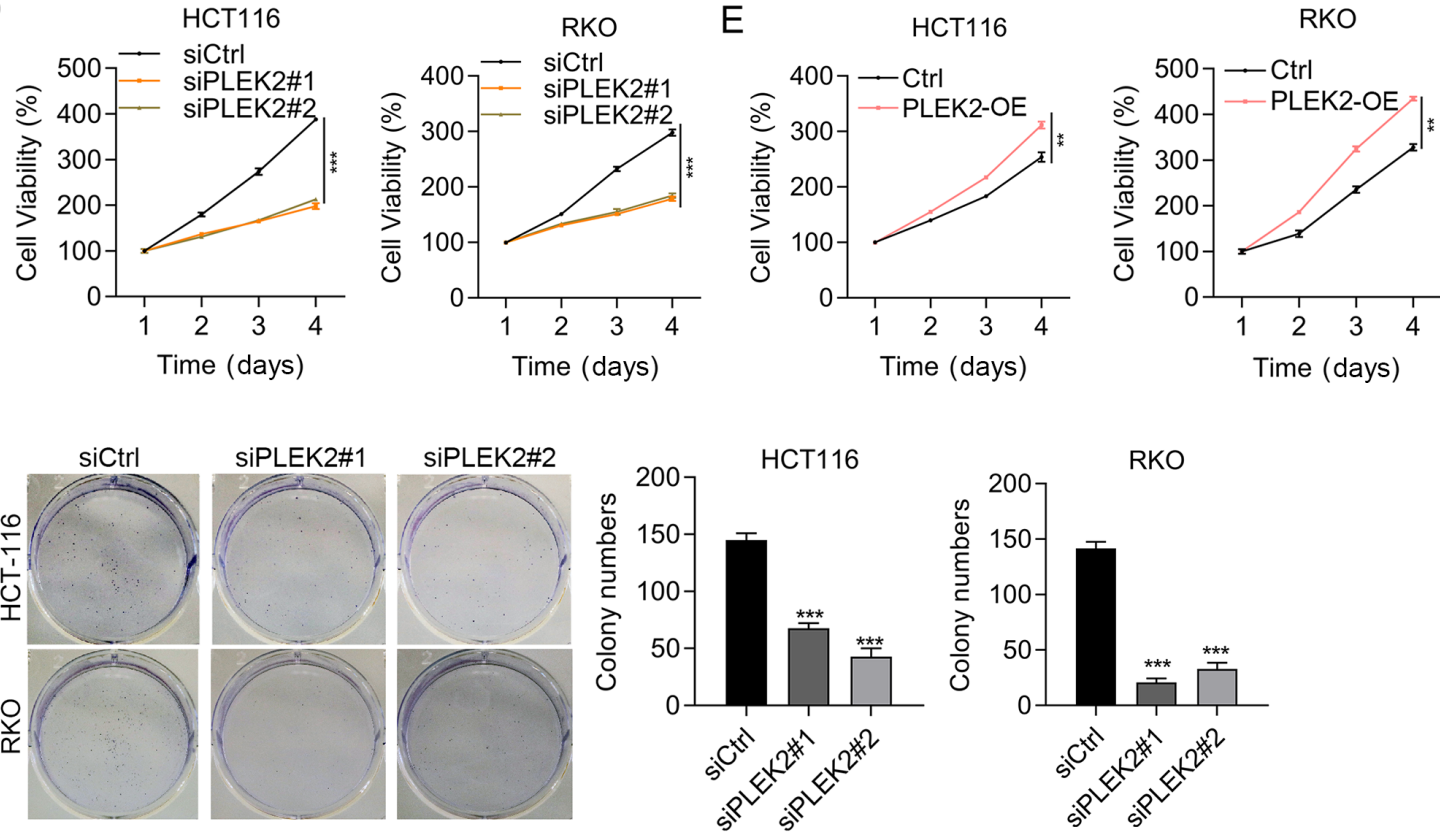

G

Ctrl
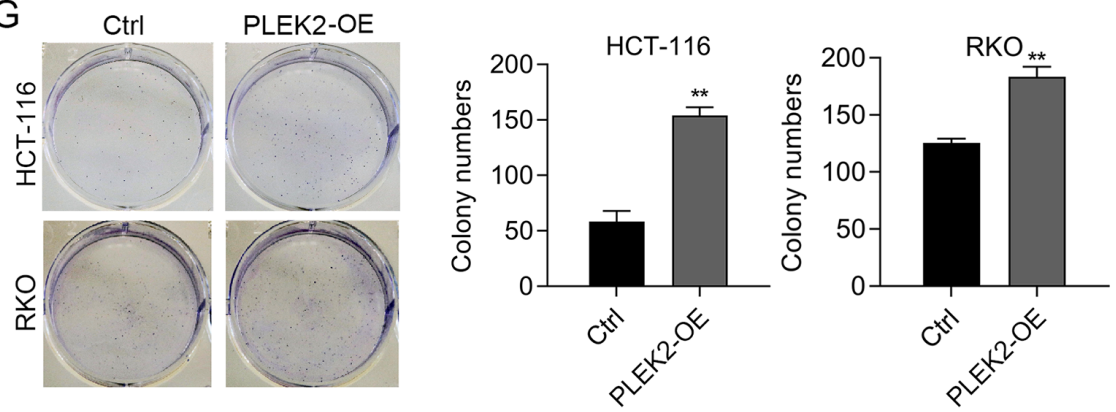

Figure 2. PLEK2 overexpression reinforces cellular functions in colorectal carcinoma cells. (A) Immunoblotting detection of PLEK2 in HIEC, RKO and HCT-116 cells. GAPDH was used as a loading control. Immunoblotting results of PLEK2 in (B) HCT116 and RKO cells transfected with siCtrl, siPLEK2\#1 and siPLEK2\#2, and (C) in Ctrl- and PLEK2-OE HCT-116 and RKO cells. GAPDH was used as a loading control. Cell proliferation was evaluated in (D) HCT116 and RKO cells transfected with siCtrl, siPLEK2\#1 and siPLEK2\#2, and (E) in Ctrl- and PLEK2-OE HCT-116 and RKO cells. ${ }^{* *} \mathrm{P}<0.01$ and ${ }^{* * * *} \mathrm{P}<0.001$. Colony formation assay of (F) HCT-116 and RKO cells transfected with siCtrl, siPLEK2\#1 and siPLEK2\#2, and (G) Ctrl- and PLEK2-OE HCT-116 and RKO cells. ${ }^{* *} \mathrm{P}<0.01$ and ${ }^{* * * *} \mathrm{P}<0.001$ vs. siCtrl or Ctrl. Ctrl, empty vector; PLEK2-OE, pleckstrin 2 overexpression; si/siRNA, small interfering RNA; siCtrl, negative control siRNA.

Cell apoptosis detection. To examine cell apoptosis, CRC cells were carefully collected using EDTA-free trypsin (Thermo Fisher Scientific, Inc.). Subsequently, cells were washed with PBS and stained with PI and Annexin V using the Annexin V-FITC/PI Apoptosis Detection kit (Shanghai Yeasen Biotechnology Co., Ltd.) according to the manufacturer's instructions. Cell apoptosis was analyzed using a flow cytometry system (cytoFlex; Beckman Coulter, Inc.). Data were analyzed using Cytexpert software (version 2.4.0.28; Beckman Coulter, Inc.).

Luciferase reporter assay. Genomic DNA was extracted from HCT116 cells using genomic DNA extraction kit (cat. no. ab156900; Abcam). For binding assessment of transcrip- tion factor $\beta$-catenin to the PLEK2 gene promoter, the promoter sequence of PLEK2 was amplified by PCR assay using a Taq kit (New England Biolabs). The primers of PLEK2 were as follows: Forward, 5'-TGCCAAAGAAAATGCCACT TCTTCTAAGCCTCAG-3' and reverse, 5'-AGCTCCGAC GCGGCAGGG-3'. The thermocycling conditions were as follows: Initial denaturation at $95^{\circ} \mathrm{C}$ for $30 \mathrm{sec}$; denaturation at $95^{\circ} \mathrm{C}$ for $15 \mathrm{sec}$, annealing at $60^{\circ} \mathrm{C}$ for $30 \mathrm{sec}$ and extension at $68^{\circ} \mathrm{C}$ for $2 \mathrm{~min}$ for 39 cycles; a final extension at $68^{\circ} \mathrm{C}$ for $5 \mathrm{~min}$; and then hold at $4^{\circ} \mathrm{C}$. The PCR product was cloned into the luciferase reporter vector, psi-CHECK vector (Promega Corporation). Subsequently, the luciferase reporter vectors and siRNAs of $\beta$-catenin were transfected into RKO and HCT116 
A

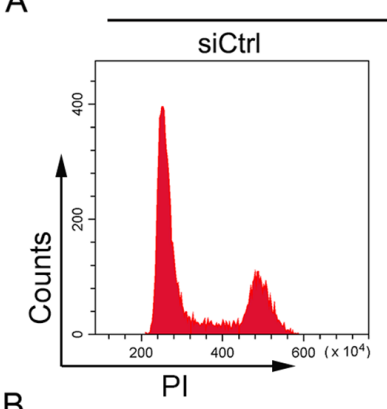

B

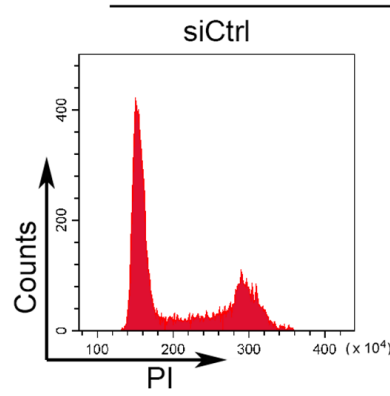

C

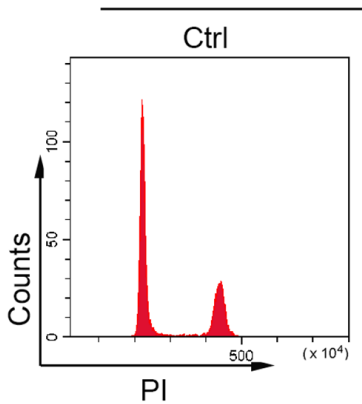

D

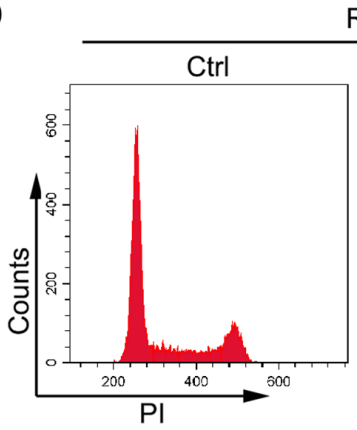

HTC-116

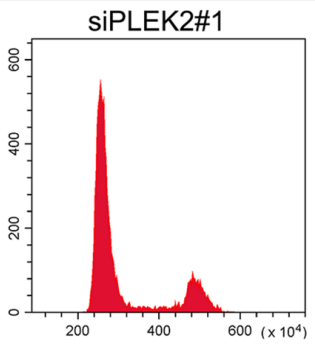

$\mathrm{RKO}$

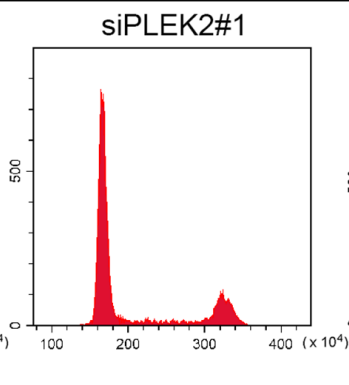

HCT-116

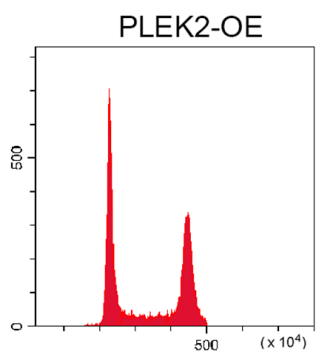

RKO

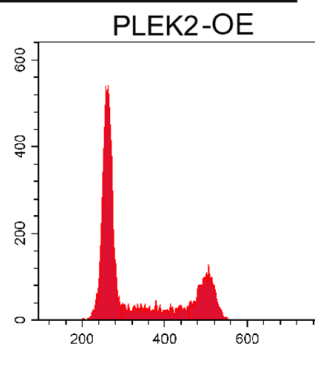

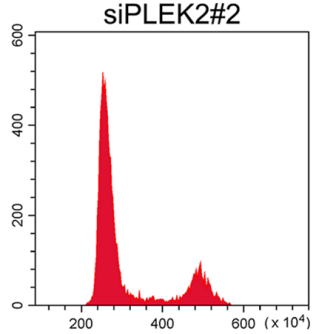

siPLEK2\#2
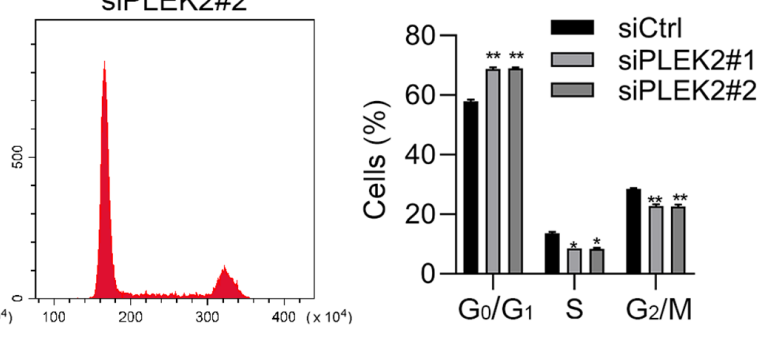

Figure 3. PLEK2 modulates cell cycle progression in colorectal carcinoma cells. (A) HCT-116 and (B) RKO cells transfected with siCtrl, siPLEK2\#1 and siPLEK2\#2 were stained with PI and the cell cycle distribution was analyzed using a flow cytometry system. Left, cell cycle graphs. Right, quantitative analysis of the cell cycle distribution. ${ }^{*} \mathrm{P}<0.05 ;{ }^{* *} \mathrm{P}<0.01$ vs. siCtrl. Ctrl and PLEK2-OE (C) HCT-116 and (D) RKO cells were stained with PI and the cell cycle distribution was analyzed using a flow cytometry system. Left, cell cycle graphs. Right, quantitative analysis of the cell cycle distribution. ${ }^{*} \mathrm{P}<0.05 ;{ }^{* *} \mathrm{P}<0.01$ vs. Ctrl. Ctrl, empty vector; PLEK2-OE, pleckstrin 2 overexpression; si/siRNA, small interfering RNA; siCtrl, negative control siRNA.

cells using Lipo2000 (Invitrogen; Thermo Fisher Scientific, Inc.). At $48 \mathrm{~h}$ post transfection, the relative luciferase activity was examined using a dual luciferase reporter assay system (Promega Corporation) according to the manufacturer's instructions. The firefly luciferase activity was normalized to Renilla luciferase activity.

Statistical analysis. Statistical data are presented as the mean \pm SEM and were analyzed using GraphPad prism software (v8.0; GraphPad Software,Inc.). The experiments were repeated at least three times. Differences between two groups were analyzed by unpaired Student's t-test. Differences among three groups were analyzed by one-way ANOVA followed by Tukey's post hoc test. Linear regression analysis was performed to analyze the Spearman correlation between PLEK2 and $\beta$-catenin using the GEPIA2021 database (http://gepia.cancer-pku.cn/) (23). P $<0.05$ was considered to indicate a statistically significant difference.

\section{Results}

APC/ $\beta$-catenin upregulates PLEK2 in CRC cells and patients with $C R C$. To determine whether APC/ $\beta$-catenin regulates 
A

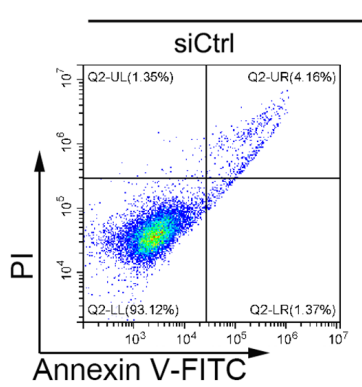

B

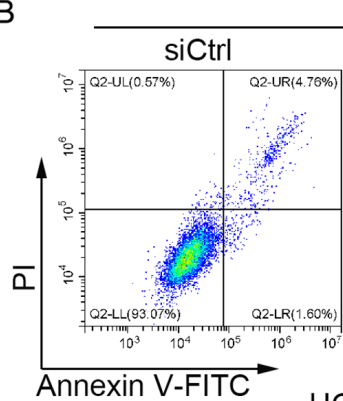

HTC-116

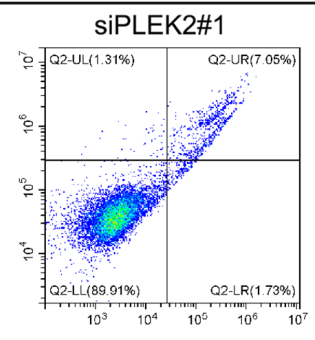

RKO

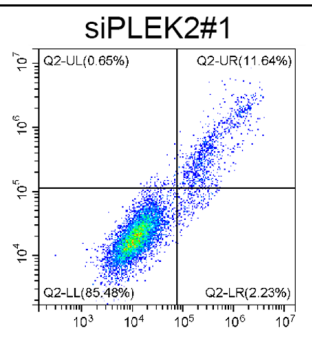

HCT-116
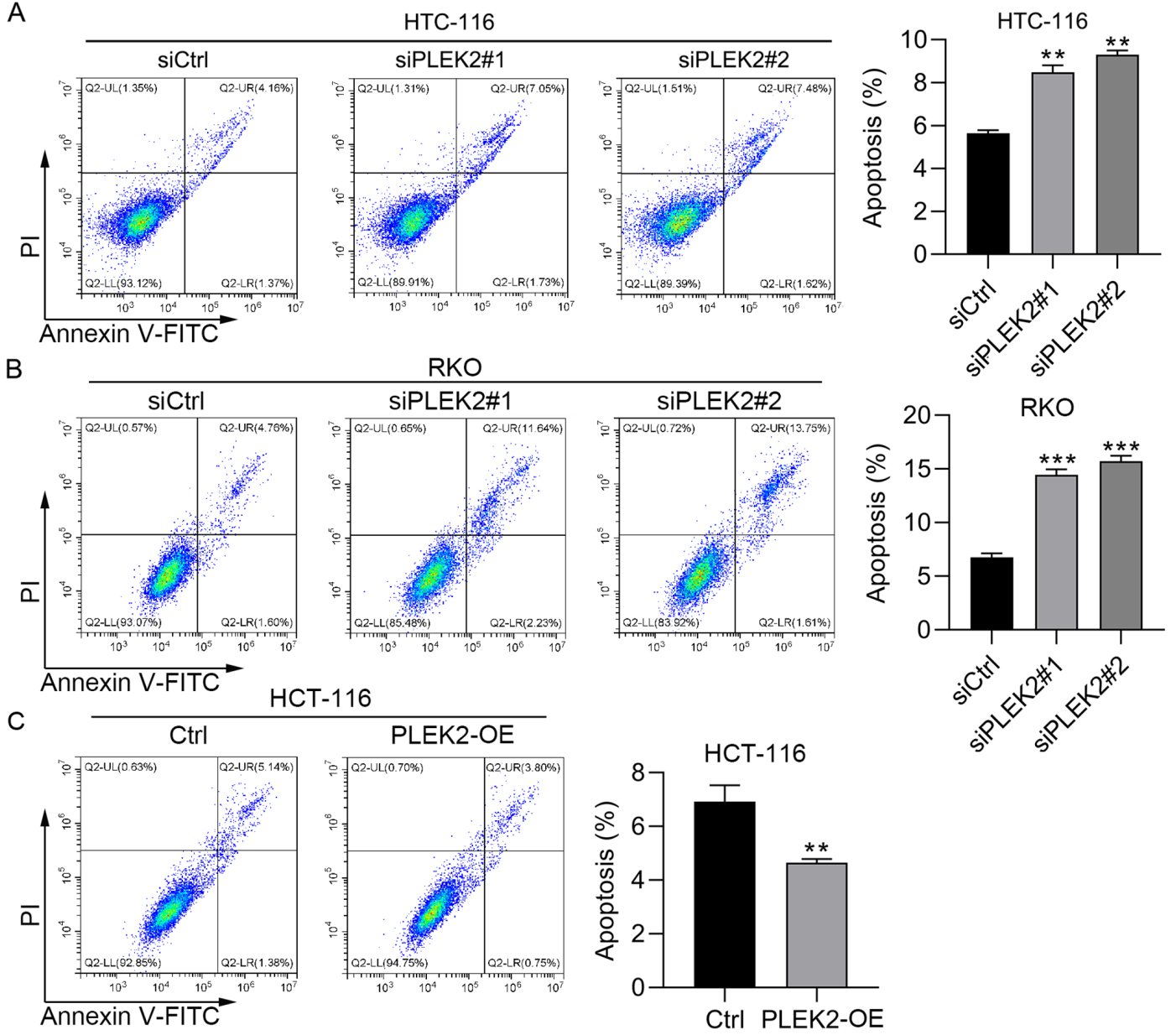

D

RKO
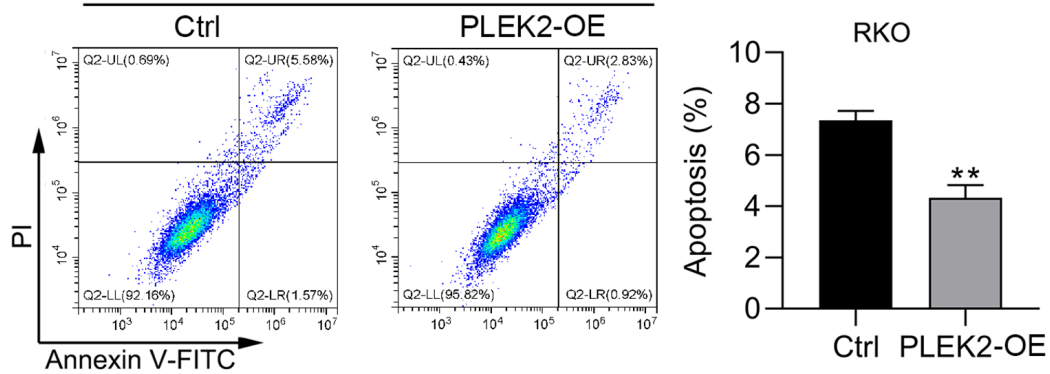

Figure 4. PLEK2 modulates apoptosis in colorectal carcinoma cells. (A) HCT-116 and (B) RKO cells transfected with siCtrl, siPLEK2\#1 and siPLEK2\#2 were stained with PI/Annexin V-FITC and cell apoptosis was analyzed using a flow cytometry system. Left, apoptosis graphs. Right, quantitative analysis of cell apoptosis. Ctrl and PLEK2-OE (C) HCT-116 and (D) RKO cells were stained with PI/Annexin V-FITC and cell apoptosis was analyzed using a flow cytometry system. Left, apoptosis graphs. Right, quantitative analysis of cell apoptosis. ${ }^{* *} \mathrm{P}<0.01 ;{ }^{* * * *} \mathrm{P}<0.001$ vs. siCtrl or Ctrl. Ctrl, emptry vector; PLEK2-OE, pleckstrin 2 overexpression; si/siRNA, small interfering RNA; siCtrl, negative control siRNA.

PLEK2 in CRC cells, APC was knocked down in HCT-116 cells. Immunoblotting results demonstrated that APC was efficiently silenced after transfection of HCT-116 cells with siRNAs against APC (Fig. 1A). In addition, APC knockdown stimulated PLEK2 expression at both the mRNA and protein levels (Fig. 1A-C). Furthermore, knockdown of APC did not increase the total $\beta$-catenin expression but increased its nuclear translocation (Fig. 1A and B). To investigate whether APC regulation of PLEK2 was dependent on upregulation of $\beta$-catenin, CTNNB1, which encodes $\beta$-catenin, was further knocked down in APC-silenced HCT-116 cells. $\beta$-catenin was effectively knocked down using siRNA as detected by RT-qPCR (Fig. S1). The western blotting results suggested that upregulation of PLEK2 was reduced after silencing of $\beta$-catenin (Fig. 1A). Consistent results were observed in APC-knockdown HCT-116 cells (Fig. 1C). To check whether $\beta$-catenin modulates the transcription activity of PLEK2, a dual-luciferase activity assay was performed and revealed that downregulation of $\beta$-catenin reduced the luciferase activity compared with the control group (Fig. 1D). These results suggested that $\beta$-catenin binds to the promoter sequence of PLEK 2 to activate APC $/ \beta$-catenin and then upregulate PLEK2 at the transcriptional level.

Subsequently, the present study aimed to explore the clinical relevance of APC and PLEK2 in CRC. First, the expression levels of PLEK2 in patients with CRC were explored using 
A

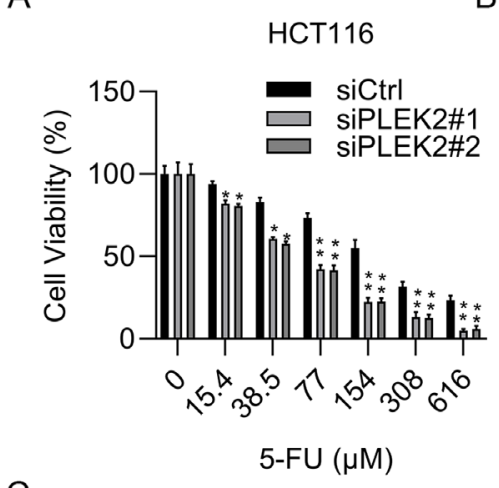

C

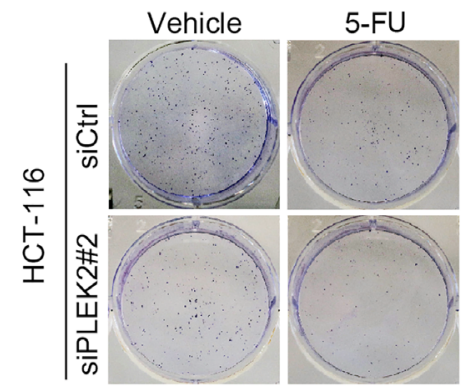

D

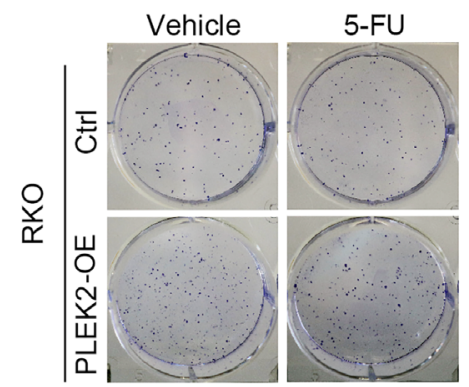

B
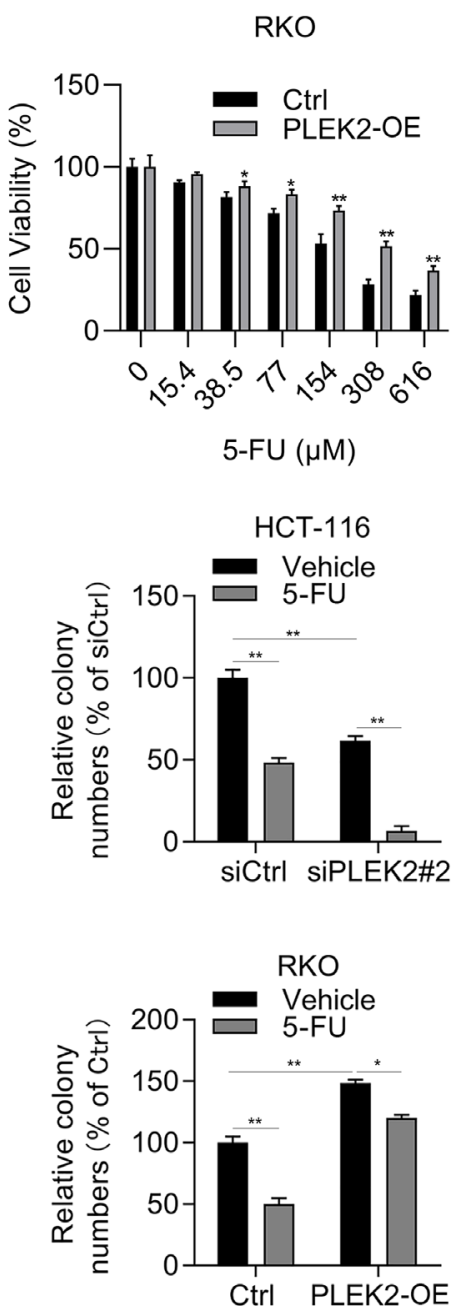

Figure 5. PLEK2 expression predicts the sensitivity of colorectal carcinoma cells to 5-FU. (A) HCT-116 cells transfected with siCtrl, siPLEK2\#1 and siPLEK2\#2 were incubated with various concentrations of $5-\mathrm{FU}$ for $48 \mathrm{~h}$. Cell viability was assessed by $\mathrm{CCK} 8$ analysis. ${ }^{*} \mathrm{P}<0.05 ;^{* *} \mathrm{P}<0.01 \mathrm{vs}$. siCtrl. $(\mathrm{B}) \mathrm{Ctrl}$ and PLEK2-OE RKO cells were incubated with various concentrations of 5 -FU for $48 \mathrm{~h}$. Cell proliferation was assessed by $\mathrm{CCK} 8$ analysis. ${ }^{*} \mathrm{P}<0.05 ;{ }^{* *} \mathrm{P}<0.01$ vs. Ctrl. (C) siCtrl and siPLEK2\#2 HCT-116 cells were seeded in 6-well plates at an equal density and incubated with vehicle DMSO or 5-FU for 10 days. Subsequently, colony formation was analyzed. ${ }^{* *} \mathrm{P}<0.01$ vs. vehicle. (D) Ctrl and PLEK2-OE RKO cells were seeded in 6-well plates at an equal density and incubated with vehicle or 5-FU for 10 days. Subsequently, colony formation was analyzed. * $\mathrm{P}<0.05$ and ${ }^{* *} \mathrm{P}<0.01$ vs. vehicle. 5-FU, 5 -fluorouracil; CCK8, Cell Counting Kit-8; Ctrl, empty vector; PLEK2-OE, pleckstrin 2 overexpressing; si/siRNA, small interfering RNA; siCtrl, negative control siRNA.

TCGA. A total of 257 CRC tissues and 349 normal tissues were included in the analysis. The results demonstrated that PLEK2 was significantly upregulated in CRC tissues compared with normal tissues (Fig. 1E). Furthermore, APC expression was inversely associated with PLEK2 expression in patients with CRC (Fig. 1F). Therefore, negative regulation of PLEK 2 by APC may exist in patients with CRC.

PLEK2 promotes CRC cell proliferation. The present study next assessed whether PLEK2 modulated CRC cell function by assessing cell proliferation and growth. To this aim, the present study firstly examined the basic expression levels of PLEK2 in colorectal normal cells (HIEC) and CRC cell lines (RKO and HCT-116). PLEK2 was highly expressed in RKO and HCT-116 cells compared with HIEC cells (Fig. 2A). PLEK2 was knocked down and overexpressed in HCT-116 and RKO cells, respectively. Immunoblotting results validated the knockdown and overexpression efficiency (Fig. 2B and C). As indicated by CCK 8 assay results, PLEK 2 knockdown reduced the proliferation of HCT-116 and RKO cells, while PLEK2 overexpression promoted both HCT-116 and RKO cell proliferation (Fig. 2D and E). In addition, the colony formation of CRC cells was suppressed by PLEK2 knockdown and promoted by PLEK2 overexpression (Fig. 2F and G). Therefore, PLEK2 upregulation in CRC may serve tumor-promoting roles.

PLEK2 regulates the cell cycle and apoptosis in CRC cells. To further determine the function of PLEK2, PI staining and $\mathrm{PI} /$ Annexin V staining were performed to detect the cell cycle distribution and apoptosis in CRC cells with PLEK2 knockdown or overexpression. Based on PI staining analysis of the cell cycle distribution using flow cytometry, PLEK2 knockdown promoted HCT-116 and RKO cell cycle arrest at $\mathrm{G}_{0} / \mathrm{G}_{1}$ phase, while overexpression of PLEK2 decreased the CRC cell percentage at $G_{0} / G_{1}$ phase (Fig. 3A-D). PI/Annexin $V$ staining results demonstrated that PLEK2 knockdown induced apoptosis in HCT-116 and RKO cells (Fig. 4A and B). Furthermore, overexpression of PLEK2 suppressed HCT-116 and RKO cell 
apoptosis (Fig. 4C and D). Collectively, PLEK2 suppressed cell apoptosis and promoted cell cycle progression in CRC.

PLEK2 overexpression confers 5-FU resistance in CRC cells. Chemotherapy resistance is a clinical problem for patients with CRC. 5-FU represents the most commonly used chemotherapy drug for patients with CRC (26). Therefore, the present study next attempted to investigate whether PLEK2 regulated the sensitivity of CRC cells to 5-FU. Firstly, CRC cells were incubated with different concentrations of 5-Fu for $48 \mathrm{~h}$. The results demonstrated that PLEK2 knockdown enhanced the cytotoxicity of 5-FU in HCT-116 cells (Fig. 5A), while overexpression of PLEK2 decreased the cytotoxicity of 5-FU in RKO cells (Fig. 5B). Since the cell viability assay results demonstrated that 5-FU inhibited cell proliferation at $15.4 \mu \mathrm{M}$ in HCT-116 cells and at $38.5 \mu \mathrm{M}$ in RKO cells, respectively, these concentrations were selected for subsequent experiments. Similarly, a colony formation assay indicated that downregulation of PLEK2 obviously enhanced the anti-growth effect of 5-FU at $15.4 \mu \mathrm{M}$ in HCT-116 cells, while PLEK2 overexpression significantly reduced the toxicity of 5-FU at $38.5 \mu \mathrm{M}$ in RKO cells (Fig. 5C and D). Overall, PLEK2 promoted 5-FU resistance in CRC cells.

\section{Discussion}

The present study demonstrated that upregulation of PLEK2 not only acted as an oncogene activated by APC/ $\beta$-catenin signaling at the transcriptional level but also as a predictor for 5-FU resistance in CRC. Based on knockdown and overexpression results, PLEK2 contributed to the proliferation of CRC cells. PLEK2 also regulated cell cycle progression and cell apoptosis. Furthermore, PLEK2 promoted 5-FU resistance in CRC cells. The present study demonstrated that APC/ $\beta$-catenin upregulation of PLEK 2 contributes to CRC cell proliferation and 5-FU resistance.

Abundant evidence has demonstrated that the tumor suppressor gene APC is commonly mutated or absent in CRC (27-29). Inactivation of APC promotes the protein stability of $\beta$-catenin by regulating GSK3 $\beta$-mediated phosphorylation of $\beta$-catenin, resulting in activation of the $\mathrm{Wnt} / \beta$-catenin signaling pathway (30). Stable $\beta$-catenin then enters into the cell nucleus and exerts transcription factor functions. Numerous targets, including guanine nucleotide-binding protein, axin 2 , leucine rich repeat containing $\mathrm{G}$ protein-coupled receptor 5 and NKD inhibitor of WNT signaling pathway, are activated by $\beta$-catenin during the development of cancer (31). During the past years, large amounts of effort have been focused on the exploration of small molecule inhibitors to specifically target the activity of the Wnt/ $\beta$-catenin signaling cascade (14). However, the side effects, such as tissue homeostasis and regeneration impairment, caused by these inhibitors limit their application for the treatment of patients with CRC (26). Therefore, it is urgent to identify novel downstream effectors of Wnt/ $\beta$-catenin to enable the development of effective drugs with limited side effects. The present study revealed that knockdown of APC promoted $\beta$-catenin and PLEK2 expression. Inhibition of $\beta$-catenin downregulated PLEK2 expression in CRC cells at both the mRNA and protein levels. Notably, As APC promoted $\beta$-catenin through regulating GSK3 $\beta$-mediated phosphorylation (25), the present study demonstrated that $\beta$-catenin directly regulated the transcription activity of PLEK2 promoter as demonstrated by a dual luciferase activity assay. Thus, these results demonstrated that APC suppressed the expression of PLEK2 and $\beta$-catenin.

PLEK2 is a paralog of pleckstrin that can bind to polyphosphoinositides and regulate actin rearrangement $(16,32,33)$. Dysregulation of PLEK2 is involved in the development of different cancer types $(17,34)$. For instance, transcriptome analysis has demonstrated that PLEK2 is highly expressed in the whole blood cells from patients with melanoma (35). In MPNs, depletion of PLEK2 alleviates JAK2V617F-triggered malignant phenotypes in a transgenic mouse model (21). PLEK2 expression is upregulated in patients with GBC, whereas overexpression of PLEK2 promotes GBC carcinogenesis by potentiating PLEK2/EGFR signaling (17). In addition, PLEK2 is highly expressed in lung adenocarcinoma samples and associated with poor prognosis of the patients (34). Upregulation of PLEK2 also serves a tumor-promoting role in NSCLC via regulation of the ubiquitination-dependent stabilization of SH2 domain containing inositol 5-phosphatase 2 (22). Although PLEK2 is upregulated with activation of APC/ $\beta$-catenin signaling in CRC cells and patients with $\mathrm{CRC}$, to the best of our knowledge, the role of PLEK2 in CRC remains undetermined. The present study demonstrated that PLEK2 overexpression resulted in enhanced cell cycle progression, reduced cell apoptosis and increased cell proliferation. Furthermore, PLEK2 overexpression conferred resistance to 5-FU in CRC cells. This suggested that PLEK2 serves an oncogenic role in CRC.

In conclusion, the present study demonstrated that PLEK2 served as a downstream substrate of the APC/ $\beta$-catenin signaling cascade. Overexpression of PLEK2 contributed to the proliferation of CRC cells. Furthermore, PLEK2 was associated with the sensitivity to 5-FU treatment in CRC cells. Therefore, APC/ $\beta$-catenin activation of PLEK2 may contribute to $\mathrm{CRC}$ growth and chemotherapy resistance.

\section{Acknowledgements}

Not applicable.

\section{Funding}

The present study was financially supported by Shanxi Natural Science Foundation (grant nos. 201701D221176 and 201901D111413), The Health and Family Planning Commission of Shanxi Province Scientific Research Project (grant no. 201601032), and The Doctor Start-up Fund of The First Hospital of Shanxi Medical University (grant no. YB161705).

\section{Availability of data and materials}

The datasets used and/or analyzed during the current study are available from the corresponding author on reasonable request.

\section{Authors' contributions}

YG designed the present study. BC carried out most of the experiments. NZ and JJ performed quantitative PCR analysis. 
$\mathrm{ZZ}$ and MP performed western blot analysis. KJ, YS and XC analyzed and interpretated data. YG and BC confirmed the authenticity of all the raw data. All authors read and approved the final manuscript.

\section{Ethics approval and consent to participate}

Not applicable.

\section{Patient consent for publication}

Not applicable.

\section{Competing interests}

The authors declare that they have no competing interests.

\section{References}

1. No authors listed: Erratum: Global cancer statistics 2018: GLOBOCAN estimates of incidence and mortality worldwide for 36 cancers in 185 countries. CA Cancer J Clin 70: 313, 2020. Erratum for: CA Cancer J Clin 68: 394-424, 2018.

2. Dekker E, Tanis PJ, Vleugels JLA, Kasi PM and Wallace MB: Colorectal cancer. Lancet 394: 1467-1480, 2019.

3. Meyerhardt JA and Mayer RJ: Systemic therapy for colorectal cancer. N Engl J Med 352: 476-487, 2005.

4. Gustavsson B, Carlsson G, Machover D, Petrelli N, Roth A, Schmoll HJ, Tveit KM and Gibson F: A review of the evolution of systemic chemotherapy in the management of colorectal cancer. Clin Colorectal Cancer 14: 1-10, 2015.

5. Van der Jeught K, Xu HC, Li YJ, Lu XB and Ji G: Drug resistance and new therapies in colorectal cancer. World J Gastroenterol 24 3834-3848, 2018.

6. Rattray NJW, Charkoftaki G, Rattray Z, Hansen JE, Vasiliou V and Johnson $\mathrm{CH}$ : Environmental influences in the etiology of colorectal cancer: The premise of metabolomics. Curr Pharmacol Rep 3: 114-125, 2017.

7. Caspi M, Wittenstein A, Kazelnik M, Shor-Nareznoy Y and Rosin-Arbesfeld R: Therapeutic targeting of the oncogenic Wnt signaling pathway for treating colorectal cancer and other colonic disorders. Adv Drug Deliv Rev 169: 118-136, 2021.

8. Carethers JM and Jung BH: Genetics and Genetic Biomarkers in Sporadic Colorectal Cancer. Gastroenterology 149: 1177-1190.e3, 2015.

9. Shang $\mathrm{S}$, Hua F and $\mathrm{Hu} \mathrm{ZW}$ : The regulation of $\beta$-catenin activity and function in cancer: Therapeutic opportunities. Oncotarget 8 : 33972-33989, 2017.

10. Bian J, Dannappel M, Wan C and Firestein R: Transcriptional Regulation of Wnt/ $\beta$-Catenin Pathway in Colorectal Cancer. Cells 9: 2125, 2020.

11. Cho YH, Ro EJ, Yoon JS, Mizutani T, Kang DW, Park JC, Il Kim T, Clevers $\mathrm{H}$ and Choi KY: 5-FU promotes stemness of colorectal cancer via p53-mediated WNT/ $\beta$-catenin pathway activation. Nat Commun 11: 5321, 2020.

12. Li Y, Wang R, Huang D, Ma X, Mo S, Guo Q, Fu G, Li Y, Xu X, $\mathrm{Hu} \mathrm{X}$, et al: A novel human colon signet-ring cell carcinoma organoid line: Establishment, characterization and application. Carcinogenesis 41: 993-1004, 2020.

13. Jung YS and Park JI: Wnt signaling in cancer: Therapeutic targeting of Wnt signaling beyond $\beta$-catenin and the destruction complex. Exp Mol Med 52: 183-191, 2020.

14. Krishnamurthy Nand Kurzrock R: Targeting the Wnt/beta-catenin pathway in cancer: Update on effectors and inhibitors. Cancer Treat Rev 62: 50-60, 2018.

15. Zhan T, Rindtorff $\mathrm{N}$ and Boutros $\mathrm{M}$ : Wnt signaling in cancer. Oncogene 36: 1461-1473, 2017.

16. Hu MH, Bauman EM, Roll RL, Yeilding $\mathrm{N}$ and Abrams CS: Pleckstrin 2, a widely expressed paralog of pleckstrin involved in actin rearrangement. J Biol Chem 274: 21515-21518, 1999.

17. Shen H, He M, Lin R, Zhan M, Xu S, Huang X, Xu C, Chen W, Yao Y, Mohan M and Wang J: PLEK2 promotes gallbladder cancer invasion and metastasis through EGFR/CCL2 pathway. J Exp Clin Cancer Res 38: 247, 2019.
18. Wang J, He Z, Sun B, Huang W, Xiang J, Chen Z, Li Z and Gu X: Pleckstrin-2 as a Prognostic Factor and Mediator of Gastric Cancer Progression. Gastroenterol Res Pract 2021: 5527387, 2021.

19. Wang J, Sun Z, Wang J, Tian Q, Huang R, Wang H, Wang X and Han F: Expression and prognostic potential of PLEK2 in head and neck squamous cell carcinoma based on bioinformatics analysis. Cancer Med 10: 6515-6533, 2021.

20. Cacemiro MDC, Cominal JG, Tognon R, Nunes NS, Simões BP, Figueiredo-Pontes LL, Catto LFB, Traina F, Souto EX, Zambuzi FA, et al: Philadelphia-negative myeloproliferative neoplasms as disorders marked by cy tokine modulation. Hematol Transfus Cell Ther 40: 120-131, 2018. Erratum in: Hematol Transfus Cell Ther 43: 117, 2021.

21. Zhao B, Mei Y, Cao L, Zhang J, Sumagin R, Yang J, Gao J, Schipma MJ, Wang Y, Thorsheim C, et al: Loss of pleckstrin-2 reverts lethality and vascular occlusions in JAK2V617F-positive myeloproliferative neoplasms. J Clin Invest 128: 125-140, 2018.

22. Wu DM, Deng SH, Zhou J, Han R, Liu T, Zhang T, Li J, Chen JP and Xu Y: PLEK2 mediates metastasis and vascular invasion via the ubiquitin-dependent degradation of SHIP2 in non-small cell lung cancer. Int J Cancer 146: 2563-2575, 2020. Erratum in: Int J Cancer 149: E11, 2021.

23. Weinstein JN, Collisson EA, Mills GB, Shaw KR, Ozenberger BA, Ellrott K, Shmulevich I, Sander C and Stuart JM; Cancer Genome Atlas Research Network: The Cancer Genome Atlas Pan-Cancer analysis project. Nat Genet 45: 1113-1120, 2013.

24. Livak KJ and Schmittgen TD: Analysis of relative gene expression data using real-time quantitative PCR and the 2(-Delta Delta C(T)) Method. Methods 25: 402-408, 2001.

25. Chai B, Guo Y, Cui X, Liu J, Suo Y, Dou Z and Li N: MiR-223-3p promotes the proliferation, invasion and migration of colon cancer cells by negative regulating PRDM1. Am J Transl Res 11: 4516-4523, 2019.

26. Xie YH, Chen YX and Fang JY: Comprehensive review of targeted therapy for colorectal cancer. Signal Transduct Target Ther 5: 22, 2020.

27. Michels BE, Mosa MH, Streibl BI, Zhan T, Menche C, Abou-El-Ardat K, Darvishi T, Członka E, Wagner S, Winter J, et al: Pooled In Vitro and In Vivo CRISPR-Cas9 Screening Identifies Tumor Suppressors in Human Colon Organoids. Cell Stem Cell 26: 782-792.e7, 2020.

28. Ragusa S, Prat-Luri B, González-Loyola A, Nassiri S, Squadrito ML, Guichard A, Cavin S, Gjorevski N, Barras D, Marra G, et al: Antiangiogenic immunotherapy suppresses desmoplastic and chemoresistant intestinal tumors in mice. J Clin Invest 130: 1199-1216, 2020.

29. Zhang L and Shay J: Multiple Roles of APC and its Therapeutic Implications in Colorectal Cancer. J Natl Cancer Inst 109: djw332, 2017.

30. Gao C, Xiao G and Hu J: Regulation of Wnt/ $\beta$-catenin signaling by posttranslational modifications. Cell Biosci 4: 13, 2014.

31. Yang B, Mao L, Li Y, Li Q, Li X, Zhang Y and Zhai Z: $\beta$-catenin, leucine-rich repeat-containing $\mathrm{G}$ protein-coupled receptor 5 and GATA-binding factor 6 are associated with the normal mucosa-adenoma-adenocarcinoma sequence of colorectal tumorigenesis. Oncol Lett 15: 2287-2295, 2018.

32. Inazu T, Yamada K and Miyamoto K: Cloning and expression of pleckstrin 2, a novel member of the pleckstrin family. Biochem Biophys Res Commun 265: 87-93, 1999.

33. Hamaguchi N, Ihara S, Ohdaira T, Nagano H, Iwamatsu A, Tachikawa $\mathrm{H}$ and Fukui Y: Pleckstrin-2 selectively interacts with phosphatidylinositol 3-kinase lipid products and regulates actin organization and cell spreading. Biochem Biophys Res Commun 361: 270-275, 2007.

34. Zhang W, Li T, Hu B and Li H: PLEK2 Gene Upregulation Might Independently Predict Shorter Progression-Free Survival in Lung Adenocarcinoma. Technol Cancer Res Treat 19: $1533033820957030,2020$.

35. Luo Y, Robinson S, Fujita J, Siconolfi L, Magidson J, Edwards CK, Wassmann K, Storm K, Norris DA, Bankaitis-Davis D, et al: Transcriptome profiling of whole blood cells identifies PLEK2 and C1QB in human melanoma. PLoS One 6: e20971, 2011.

This work is licensed under a Creative Commons Attribution-NonCommercial-NoDerivatives 4.0 International (CC BY-NC-ND 4.0) License. 